Anim. Behav., 1994, 48, 169-176

\title{
Heritability and repeatability of behavioural attributes affecting foraging success and fitness in water striders
}

\author{
WOLF U. BLANCKENHORN* $†$ \& DIRK PERNER $\$ \S$ \\ *Biology Department, Concordia University, 1455 de Maisonneuve Blvd Ouest, Montréal, Québec, \\ H3G 1 M8 Canada \\ fDepartment of Biological Sciences, State University of New York, Albany, NY 12222, U.S.A.
}

(Received 15 April 1993; initial acceptance 18 April 1993;

final acceptance 21 June 1993; MS. number: A6527R)

\begin{abstract}
Heritabilities and repeatabilities are presented for various behavioural attributes affecting foraging performance and fitness in Aquarius (Gerris) remigis (Heteroptera: Gerridae) females. These behavioural attributes were patch choice, foraging success, capture accuracy, and measures of mobility, activity, skittishness and aggressiveness. Most heritabilities were not significantly different from zero, which may be related to the low sample size. Conclusions as to the potential of direct selection on behaviour in this species were consequently limited. In contrast, with a few exceptions (capture accuracy, foraging success), most repeatabilities were significant and at times high (range $=0.22-0.79$ ), indicating consistent, stereotypical individual behaviour. The life history or reproductive state of the daughter generation individuals significantly affected the magnitude of the repeatabilities as well as the mean values of many of the variables (notably mobility and aggressiveness), the latter in a manner consistent with field observations. This indicates that the state of the organism affects the general environmental variance, thus contributing to the discrepancies between the repeatabilities and the heritabilities obtained. It is suggested that common physiological processes (e.g. hormones) may underlie several of the behavioural attributes examined, resulting in possible pleiotropic effects and constraints on selection in a heterogeneous environment. It is further suggested that field studies of selection on behavioural attributes may be a more fruitful approach in this species, whose suitability for genetic analysis is limited.
\end{abstract}

The explicit assumption underlying much of foraging theory is that foraging behaviour is shaped, directly or indirectly, by natural selection (Stephens \& Krebs 1986). This involves demonstrating (1) variation in the expression of a character, (2) fitness variation associated with that character, and (3) heritable components (Endler 1986). While demonstrating variation in the expression of a character is usually easy, showing the associated fitness variation is problematic because frequently the fitness estimators used are far detached from ideal measures like lifetime reproductive success (Blanckenhorn 1991a). Most notably, evidence for heritable components of behavioural attributes affecting foraging success

†Present address and address for reprint requests: Zoologisches Museum der Universität, Winterthurerstrasse 190, CH-8057 Zürich, Switzerland.

§Present address: Biozentrum, Zoologisches Institut II der Universität, Am Hubland, D-8700 Würzburg, Germany. and fitness is extremely scarce (see Hedrick \& Riechert 1989 for a brief review). Moreover, in most known cases genetic variation in foraging behaviour has been demonstrated between, as opposed to within, populations (Hedrick \& Riechert 1989).

In two long-term studies of the water strider, Aquarius (Gerris) remigis (Hemiptera: Gerridae), a sit-and-wait surface predator of lotic habitats, Blanckenhorn (1991a, b) found consistent differences between individuals in several behavioural attributes affecting foraging success and consequently (lifetime) fitness. These included patch choice, territoriality and aggressive behaviour. The present study was designed to demonstrate a heritable basis of these and related behavioural attributes in the same population.

As a result of their inherent flexibility, often due to learning, behavioural traits are expected to show low heritabilities; consequently, they can require prohibitively large sample sizes to be 
detected by standard methods (Boake 1989). Because of time and space constraints imposed by the behaviour and life cycle of the study animal as well as the nature of our experiment, we additionally computed repeatabilities of the various behavioural measures. Repeatability quantifies trait variation within individuals relative to that between individuals. It is a measure of how consistent or stereotypical (i.e. inflexible) a character is, and hence the degree to which it is influenced by genes, setting an upper limit to its heritability (Lessels \& Boag 1987; Boake 1989; Falconer 1989). Hence, high repeatabilities are suggestive "of, that is, necessary but not sufficient for significant non-zero heritabilities (Boake 1989).

\section{METHODS}

\section{Experimental Procedure}

We essentially replicated the experiments performed by Blanckenhorn (1991a, b). Beginning in April 1991, first we repeatedly assayed a total of 21 over-wintered reproductive females for 7 weeks. (more than half their reproductive life), and subsequently two cohorts of 21 of their daughters for 6 weeks each. The mothers were collected just prior to the experiment from a stream at the Albany, New York, Rural Cemetery. Employing a half-sibling mating design (Falconer 1989), we paired the females every 3 days in their individual $40 \times 30 \mathrm{~cm}$ holding trays with the same randomly picked male. There were seven males (sires), each paired to three females (dams), so that the two tested daughters of each female were full-siblings. The first cohort of daughters was largely reproductive (17 of 21 individuals), whereas the second cohort was entirely non-reproductive, i.e. diapausing.

We assayed the striders in $100 \times 60 \times 15 \mathrm{~cm}$ laboratory foraging arenas simulating natural stream conditions, at a constant temperature of $20^{\circ} \mathrm{C}$ and natural light schedules. Each arena exhibited two foraging patches of equal area and flow rates but differing prey availabilities. We alternately assigned the left or the right patch of each of the seven arenas as the high food patch. These patch allocations were then maintained for the entire duration of the experiment. Twice a week, we simultaneously tested a set of seven individuals singly for an experimental session of approximately $2.5 \mathrm{~h}$. Each individual was observed for a total of three 5-min periods, spaced 35 min apart, by rotating through all seven arenas three times (see Blanckenhorn 1991a; 'patch choice experiment'). We then performed a skittishness and aggression test (see variable definitions below). Finally, we always added the same competitor (one of the other females), now assaying pairs for another $2 \cdot 5-\mathrm{h}$ session in exactly the same manner as singles (see Blanckenhorn 1991b). Before observations began in either experimental session, the individuals were given ca $0.5 \mathrm{~h}$ to acclimatize. We always released the single foragers into the same patch at the same location, irrespective of patch allocation, and we always released the competitor into the patch that the original forager occupied at the time.

We dispensed prey items, live flightless Drosophila melanogaster, upstream from the foragers, every $5 \mathrm{~min}$ in the rich patch and every $35 \mathrm{~min}$ in the poor patch. The rich patch thus received a total of 21 flies and the poor patch a total of three flies per experimental session. During each 5-min observation period, we recorded (1) the location and occupation (foraging or resting) of each individual within the arena every $30 \mathrm{~s},(2)$ any agonistic interactions between the two individuals and (3) individual prey captures as a function of the strider's location in the arena. At the end of the day each individual was returned to her holding tray, where she received a number of frozen D. melanogaster equal to the difference between her total foraging success and that of the most successful forager in order to keep food intake constant for all individuals. (We did not monitor whether the food was actually consumed.) For further details about set-up and methods see Blanckenhorn (1991a; 'patch choice experiment') regarding singles and (199lb) regarding pairs.

\section{Data Analysis}

The standardized set-up and methodology allowed comparisons of all behaviour assessed within and across individuals and cohorts. For each individual we calculated weekly means of six 5-min observation periods of all dependent variables, defined as follows. (1) Rich-patch occupancy (singles): proportion of time spent striding (foraging) on the water, i.e. not resting, in the rich patch when single; (2) foraging success: number of flies captured in both patches when single; (3) capture accuracy: number of grabs with the 
forelegs required to capture a fly when single; (4) patch switches (singles): frequency of patch switches when single; (5) mobility (singles): minimum distance (in $\mathrm{cm}$ ) moved within the arena (estimated as the sum of the distances travelled between 10 30-s point samples); (6) activity (singles): proportion of time spent foraging, i.e. not resting; (7) patch switches (pairs): same as (4) for pairs; (8) mobility (pairs): same as (5) for pairs; (9) activity (pairs): same as (6) for pairs; (10) aggressiveness: number of attacks against a standard opponent during the first minute following first contact after the subject's introduction into the arena; (11) skittishness: distance fled (in $\mathrm{cm}$ ) when disturbed by the observer in a standard manner (waving the hand back and forth once ca $20 \mathrm{~cm}$ above the individual).

We computed heritabilities $( \pm \mathrm{SE}$ ) from the half-sibling analysis as described in detail in Falconer (1989) and Becker (1984, page 55ff.). Sire and dam components were computed separately; the dam component contains four times the maternal effects and all dominance variance (Becker 1984, page 58). Heritabilities were deemed significant if their $95 \%$ confidence interval did not overlap zero.

We further analysed the data as an ANOVA to calculate repeatabilities ( \pm SE) following Lessels \& Boag (1987) and Becker (1984). Repeatability is defined as $r=\left(V_{\mathrm{G}}+V_{\mathrm{Eg}}\right) / V_{\mathrm{P}}$ (Falconer 1989), where $V_{\mathrm{P}}=V_{\mathrm{G}}+V_{\mathrm{Eg}_{\mathrm{g}}}+V_{\mathrm{Es}}$ is the total phenotypic variance among individuals; $V_{\mathrm{G}}$ is the genotypic variance; $V_{E s}$ is the special environmental variance within individuals due to temporary (often random) effects; and $V_{E g}$ is the general environmental variance common to all repeated measurements within individuals due to permanent effects, which hence contributes to the between-individual component. $V_{E_{s}}$ is the variance one attempts to minimize by standardizing set-up and procedure; $V_{E_{g}}$ may include environmental effects induced during development that are permanent, or global changes in the physiological state (e.g. diapause status).

For the 21 mothers there were six repeated weekly means, and for the two groups of daughters there were five repeated weekly means per variable. In all cases we excluded the data of the first week of testing to ensure a minimum degree of familiarity with the set-up on the part of the individuals. Because of the completely balanced design in all cases, we could directly compute repeatabilities using the $F$-ratio of the ANOVA (appendix 2 in Lessels \& Boag 1987): $r=(F-1) /$ $\left(F-1+n_{0}\right)$, where $n_{0}$ is the number of repeated measures, i.e. six and five for mothers and daughters, respectively.

We compared the mean scores and repeatabilities of the reproductive and non-reproductive daughters of each female directly using paired $t$-tests. Because we were interested in behavioural differences related to reproductive state, we reduced the sample size of the non-reproductive cohort to $N=17$, retaining only those individuals that could be paired with a reproductive sister of the first cohort. We used the SE formula given by Becker (1984, page 39) for the $t$-tests comparing repeatabilities. Lastly, we computed the phenotypic correlations and mean values (data square root $\left(x_{i}\right)$-transformed) of all the behavioural variables examined.

\section{RESULTS}

Heritabilities of four of 11 measures yielded significance, three in the sire component and one in the dam component; in no case were both sire and dam components significant (Table I). Heritabilities of all other variables were not different from zero.

Repeatabilities for most measures were significant and at times high (Table I), indicating consistent behaviour of the foragers in all cohorts. Capture accuracy featured the lowest numbers. Repeatabilities for the non-reproductive (diapausing) daughter cohort were consistently lower than those for the reproductive cohort. Even though paired $t$-tests in no case yielded significance (Table I), for nine of 11 variables the former repeatability was lower, a significant result according to a one-tailed binomial test $(P=0.033)$.

Table II shows the corresponding variable means. Reproductive and non-reproductive daughters differed significantly in several measures. Furthermore, within each cohort, patch switches, activity, mobility and aggressiveness (variables 4-10), both for singles and pairs, displayed high phenotypic correlations; correlations of these variables with the remaining variables, as well as correlations among the remaining variables, were inconsistent (Table III). Because of the nature of the experiment, foraging success naturally correlated highly with both activity and rich 
Table I. Heritabilities $\pm \mathrm{SB}$ of various behavioural attributes as well as their repeatablities \pm SE ( $F$-ratio; Bonferroni-adjusted $P$ ) in mothers, and in a reproductive and a non-reproductive cohort of their daughters

\begin{tabular}{|c|c|c|c|c|c|c|}
\hline \multirow[b]{2}{*}{ Variable } & \multicolumn{2}{|c|}{ Heritability component } & \multirow[b]{2}{*}{ Mothers $(N=21 ; k=6)$} & \multicolumn{2}{|c|}{ Repeatability. } & \multirow[b]{2}{*}{$t$} \\
\hline & Sire & Dam & & $\begin{array}{c}\text { Reproductive } \\
\text { F, }(N=17 ; k=5)\end{array}$ & $\begin{array}{cc}\text { Non-reproductive } \\
\mathrm{F}_{1}(N=17 ; k=5)\end{array}$ & \\
\hline Rich-patch occupancy & $1.52 \pm 0.69^{*}$ & $0.33 \pm 0.29$ & $0.23 \pm 0.10(2.83 ;<0.01)$ & $0.79 \pm 0.07(20.36 ;<0.01)$ & $0.58 \pm 0.11(8.04 ;<0.01)$ & 1.56 \\
\hline Foraging success & $0.95 \pm 0.51^{*}$ & $-0.50 \pm 0.46$ & $0.07 \pm 0.07(1.45 ; 0.75)$ & $0.52 \pm 0.12(6.36 ;<0.01)$ & $0.30 \pm 0.13(3.14 ; 0.01)$ & $1 \cdot 26$ \\
\hline Capture accuracy & $0.33 \pm 0.31$ & $0.25 \pm 1.06$ & $0.11 \pm 0.08(1.77 ; 0.36)$ & $0.22 \pm 0.12 \quad(2.41 ; 0.08)$ & $0.03 \pm 0.09(1.14 ; 0.99)$ & $1 \cdot 28$ \\
\hline Patch switches (single) & $0.58 \pm 0.50$ & $-1.09 \pm 0.78$ & $0.29 \pm 0.10(3.46 ;<0.01)$ & $0.32 \pm 0.13 \quad(3.32 ;<0.01)$ & $0.25 \pm 0.12(2.69 ; 0.04)$ & 0.40 \\
\hline Mobility (single) & $0.66 \pm 0.52$ & $-1.29 \pm 0.77$ & $0.41 \pm 0.10(5.14 ;<0.01)$ & $0.26 \pm 0.12 \quad(2.75 ; 0.03)$ & $0.22 \pm 0.12(2.42 ; 0.08)$ & 0.26 \\
\hline Activity (single) & $0.85 \pm 0.74$ & $0.24 \pm 0.74$ & $0.29 \pm 0.10(3.44 ;<0.01)$ & $0.34 \pm 0.13 \quad(3.63 ;<0.01)$ & $0.33 \pm 0.13(3.51 ;<0.01)$ & 0.06 \\
\hline Patch switches (pairs) & $0.57 \pm 0.48$ & $-0.31 \pm 0.79$ & $0.29 \pm 0.10(3.47 ;<0.01)$ & $0.34 \pm 0.13 \quad(3.58 ;<0.01)$ & $0.22 \pm 0.12(2.39 ; 0.08)$ & 0.67 \\
\hline Mobility (pairs) & $0.83 \pm 0.54$ & $-1.81 \pm 0.77$ & $0.52 \pm 0.10(7.40 ;<0.01)$ & $0.29 \pm 0.13 \quad(3.04 ; 0.01)$ & $0.28 \pm 0.12(2.84 ; 0.01)$ & 0.28 \\
\hline Activity (pairs) & $0.28 \pm 0.51$ & $0.15 \pm 0.86$ & $0.48 \pm 0.10(6.43 ;<0.01)$ & $0.30 \pm 0.13 \quad(3.17 ; 0.01)$ & $0.44 \pm 0.13(4.95 ;<0.01)$ & -0.79 \\
\hline Aggressiveness & $-0.09 \pm 0.38$ & $-0.02 \pm 0.94$ & $0.45 \pm 0.10(5.87 ;<0.01)$ & $0.44 \pm 0.13 \quad(4.85 ;<0.01)$ & $0.34 \pm 0.13(3.61 ;<0.01)$ & 0.56 \\
\hline Skittishness & $-0.26 \pm 0.68$ & $0.79 \pm 0.42^{*}$ & $0.26 \pm 0.10(3.10 ;<0.01)$ & $0.37 \pm 0.13 \quad(3.89 ;<0.01)$ & $0.37 \pm 0.13(3.91 ;<0.01)$ & 0.00 \\
\hline Body length & $-0.08 \pm 0.31$ & $-0.75 \pm 0.92$ & & & & \\
\hline
\end{tabular}

\section{* $p<0.05$.}

+Comparison of the repeatabilities of the two daughter cohorts. 
Table II. Variable means ( \pm SD) of mothers, and of a reproductive and a non-reproductive cohort of their daughters

\begin{tabular}{|c|c|c|c|c|}
\hline Variable & Mothers $(N=2 \mathrm{l})$ & $\begin{array}{c}\text { Reproductive } \\
\mathrm{F}_{1}(N=17)\end{array}$ & $\begin{array}{c}\text { Non-reproductive } \\
\mathrm{F}_{1}(N=17)\end{array}$ & $t ; P \uparrow$ \\
\hline Rich-patch occupancy (proportion) & $0.68 \pm 0.17$ & $0.65 \pm 0.37$ & $0.53 \pm 0.35$ & $0.84 ; 0.997$ \\
\hline Foraging success (number of flies) & $7 \cdot 80 \pm 1 \cdot 11$ & $7 \cdot 46 \pm 3.20$ & $3.97 \pm 2.03$ & $3.72 ; 0.011$ \\
\hline Capture accuracy (number of grabs) & $2 \cdot 10 \pm 0.25$ & $2.37 \pm 0.40$ & $2.52 \pm 0.44$ & $-1.00 ; 0.987$ \\
\hline Patch switches, single (number) & $1.94 \pm 1.23$ & $0.71 \pm 0.79$ & $0.31 \pm 0.77$ & $1.48 ; 0.833$ \\
\hline Mobility, single (cm) & $148 \cdot 82 \pm 65 \cdot 51$ & $113 \cdot 50 \pm 42 \cdot 88$ & $57.61 \pm 33.04$ & $4.16 ;<0.010$ \\
\hline Activity, single (proportion) & $0.73 \pm 0.13$ & $0.80 \pm 0.21$ & $0.50 \pm 0.28$ & $3.44 ; 0.022$ \\
\hline Patch switches, pairs (number) & $2.76 \pm 1.34$ & $1.01 \pm 1.01$ & $0.38 \pm 0.26$ & $2 \cdot 55 ; 0.163$ \\
\hline Mobility, pairs (cm) & $181 \cdot 64 \pm 90.09$ & $106 \cdot 59 \pm 42 \cdot 22$ & $56 \cdot 73 \pm 25 \cdot 57$ & $4 \cdot 18 ;<0.010$ \\
\hline Activity, pairs (proportion) & $0.61 \pm 0.22$ & $0.63 \pm 0.21$ & $0.40 \pm 0.26$ & $2 \cdot 96 ; 0.064$ \\
\hline Aggressiveness (number of attacks) & $1 \cdot 27 \pm 1.00$ & $0.21 \pm 0.25$ & $0.51 \pm 0.46$ & $-2 \cdot 56 ; 0 \cdot 162$ \\
\hline Skittishness (cm fled) & $30 \cdot 63 \pm 11 \cdot 38$ & $26 \cdot 22 \pm 9 \cdot 30$ & $28 \cdot 30 \pm 11 \cdot 14$ & $-0.46 ; 0.999$ \\
\hline Body length (mm) & $13.85 \pm 0.56$ & $13.64 \pm 0.46$ & $13.54 \pm 0.60$ & $0.60 ; 0.999$ \\
\hline
\end{tabular}

tComparison of the two daughter cohorts (Bonferroni-adjusted)

patch occupancy (see also Blanckenhorn 1991a). We treated all phenotypic variables as independent in our analysis because we had no a priori reason to suspect they may be intercorrelated on physiological grounds, and because multivariate treatment may have rendered the data more difficult to interpret.

Because we made multiple comparisons, significance levels needed to be Bonferroni-adjusted: $P_{\mathrm{g}}=1-\left(1-P_{\mathrm{s}}\right)^{N}$, where $P_{\mathrm{g}}$ is the group-wise $P$-value, $P_{\mathrm{s}}$ is the individual $P$-value yielded by the analysis, and $N$ is the total number of variables compared $(N=11)$. Tables I and II present the adjusted $P$-values.

\section{DISCUSSION}

Only four of the 11 behavioural measures yielded heritabilities significantly different from zero, three in the sire component (rich-patch occupancy, foraging success, and activity when single) and one in the dam component (skittishness). Note that only the dam component includes maternal and dominance effects confounding the magnitude of the additive genetic variance. In no case were both sire and dam components significant. All other heritabilities were low and non-significant.

Low heritabilities can generally be expected for flexible behavioural traits, but in our study this may be the result of low sample size alone.
Heritabilities between 0.10 and 0.50 have been reported for simple behavioural variables (Mousseau \& Roff 1987). We may expect even lower values for the complex assessed here but ecologically more relevant behavioural variables, as behaviour necessarily implies flexible responses to environmental changes (Fagen 1982). Power analysis indicates that with seven sires we would have required $20-104$ offspring per sire, compared with the six offspring per sire achieved in our study, to detect heritabilities between 0.50 and 0.10 (respectively) with a power of 0.75 (Table 2 in Wearden 1959). Such sample sizes could not be achieved in the present study due to space constraints relating to the experimental set-up, time constraints relating to the animals' life cycle, and their relatively slow time scale of behavioural response to environmental variation (Blanckenhorn 1991a). By reducing the total period of observation per strider to 3 weeks, in our opinion the absolute minimum admissible to ensure meaningful results, we could have boosted the total sample size to at most 36 offspring per sire in one season. With hindsight, after further rearing experience with this species (W. U. Blanckenhorn, unpublished data; R. F. Preziosi, personal communication), we must concede that the suitability of this species for genetic analysis is limited. A more fruitful approach may be to assess natural selection in the field (e.g. Arnold \& Wade 1984) by linking the same behavioural attributes used here, or related ones like territoriality and 
Table III. Within-cohort phenotypic correlations of the various behavioural variables assessed $(N=17)$

\begin{tabular}{|c|c|c|c|c|c|c|c|c|c|c|c|}
\hline Variable & (1) & (2) & (3) & (4) & (5) & (6) & (7) & (8) & (9) & $(10)$ & (11) \\
\hline (1) Rich-patch occupancy & & $0.85^{* *}$ & 0.36 & 0.40 & 0.07 & $0.50^{* *}$ & $-0.50^{*}$ & 0.20 & $0 \cdot 25$ & -0.25 & 0.03 \\
\hline (2) Foraging success & $0.80^{* *}$ & & $0.49^{*}$ & $-0 \cdot 16$ & 0.34 & $0.76^{* *}$ & -0.28 & $0 \cdot 11$ & $0.62^{* *}$ & 0.04 & 0.15 . \\
\hline (3) Capture accuracy & 0.03 & 0.03 & & 0.11 & 0.20 & $0.61^{* *}$ & -0.22 & 0.11 & $0 \cdot 50^{*}$ & -0.04 & 0.09 \\
\hline (4) Patch switches, single & 0.13 & 0.32 & $0-11$ & & $0.52^{*}$ & 0.09 & $0.82 * *$ & $0.60^{* *}$ & $0 \cdot 18$ & $0 \cdot 56^{* *}$ & $0 \cdot 55^{* *}$ \\
\hline (5) Mobility, single & $0 \cdot 36$ & $0.57^{* *}$ & 0.29 & $0.77^{* *}$ & & $0.58^{* *}$ & $0.49^{*}$ & $0.86^{* *}$ & $0.62^{* *}$ & $0 \cdot 70^{* *}$ & 0.29 \\
\hline (6) Activity, single & $0.57^{* *}$ & $0.83^{* *}$ & 0.19 & $0.47^{*}$ & $0.76^{* *}$ & & 0.13 & $0.44^{*}$ & $0.89^{* *}$ & 0.23 & 0.01 \\
\hline (7) Patch switches, pairs & $0 \cdot 17$ & 0.34 & 0.00 & $0.76^{* *}$ & $0.69^{* *}$ & $0.53^{*}$ & & $0.62^{* *}$ & 0.05 & $0.59^{* *}$ & $0.49^{* *}$ \\
\hline (8) Mobility, pairs & 0.36 & $0.53^{*}$ & 0.17 & $0.69^{* *}$ & $0.88 * *$ & $0.69^{* *}$ & $0.81^{* *}$ & & $0.63^{* *}$ & $0 \cdot 80^{* *}$ & $0 \cdot 18$ \\
\hline (9) Activity, pairs & $0.52 *$ & $0.80^{* *}$ & 0.21 & 0.41 & $0.74^{* *}$ & $0.97^{* *}$ & $0.55^{*}$ & $0.72 * *$ & & $0.48^{*}$ & 0.03 \\
\hline (10) Aggressiveness & 0.28 & $0 \cdot 50^{*}$ & -0.09 & $0-20$ & 0.54 & $0.64^{*}$ & $0.62^{* *}$ & $0.73 * *$ & $0.72 * *$ & & 0.30 \\
\hline (11) Skittishness & 0.33 & 0.32 & 0.42 & 0.26 & $0.45^{*}$ & $0.52^{*}$ & 0.17 & 0.24 & 0.38 & 0.06 & \\
\hline
\end{tabular}

${ }^{*} P<0.05,{ }^{* *} P<0.01$; reproductive $\mathrm{F}_{1}$ above the diagonal; non-reproductive $\mathrm{F}_{1}$ below the diagonal. 
foraging-site tenacity, with some components of fitness (e.g. survivorship, mating success). While some of our data, gathered in another context, bear upon this question (unpublished data), we cannot at present offer comprehensive evidence in this regard. Given these arguments, the lack of significant heritable variation in the majority of behavioural attributes assayed in our study highly limits any conclusions as to the potential for direct selection on behaviour in this species.

None the less, repeatabilities of most behavjoural attributes assessed were high and significant, indicating consistent, stereotypical individual behaviour. This means that either genotypic variation in these characters is present and we just did not achieve high enough sample sizes to demonstrate it, or that there exists substantial general environmental variance. Capture accuracy had the lowest repeatabilities, probably because this measure is strongly influenced by hunger level, which can vary substantially over time. Patch choice has been shown to be influenced by learning, although this varies greatly between individuals (Blankenhorn 1991a). Furthermore, owing to the high 7:1 ratio of prey availabilites between the patches, foraging success naturally correlates highly with rich-patch occupancy. The high repeatabilities (and heritabilities) in the daughter cohorts for these two variables, which are known to change over time, are thus somewhat surprising.

By comparison, the other variables, namely patch switches, mobility, skittishness and aggressiveness, probably reflect more stable individual characteristics. Phenotypic correlations between them were high. All these measures may in fact relate to common physiological processes (e.g. hormones) that proximately affect general activity levels of individuals, as seems to be the case in some vertebrates (e.g. Tulley \& Huntingford 1989; Ketterson \& Nolan 1992). Pleiotropic effects on various behavioural attributes are a possible outcome. Selection may thus influence several behavioural (and other) traits simultaneously by acting on perhaps only a single physiological pathway (Fairbairn \& Roff 1990; Stearns et al. 1991). This may be adaptive in some situations or during some periods of an individual's life, when a particular combination of traits is favoured, but not in others. While this suggests an easy (and efficient) proximate mechanism of how selection may affect complex behavioural attributes, it may at the same time constrain the evolution of optimal behaviour in a fluctuating environment. Of course, future research needs to be directed towards identifying such potential physiological substances or pathways.

The behavioural attributes treated here are more complex than those for which genetical data have been examined in the past (see Mousseau \& Roff 1987 for a review), and are more in line with recent attempts to perform genetical studies on more ecologically relevant behaviour that has immediate fitness consequences (e.g. Bakker 1986; Hoffmann 1988; Hedrick \& Riechert 1989). The fitness impact of patch choice, activity levels, and territorial defence to a sit-and-wait predator in a foraging context has been demonstrated by Blanckenhorn (1991a, b). Mobility (including patch switches) and aggressiveness are two attributes that directly define territoriality in this and other species (Grant \& Noakes 1988; unpublished data), but their fitness impact may be complex. Some A. remigis individuals are inherently tenacious sit-and-wait predators, rarely switching patches or sites (and being less aggressive), while others are more on the move (Blanckenhorn 1991a). This may predispose them to use alternative foraging modes in the field, the relative success of which will ultimately depend on the spatio-temporal distribution of food in nature, potentially providing comparable success in a heterogeneous environment (cf. Ehlinger \& Wilson 1988). Lastly, skittishness can be viewed as a measure of an individual's ability to escape predators and hence has immediate potential fitness consequences (cf. Sih et al. 1990).

An interesting result of this study is the consistently lower repeatabilities of the nonreproductive daughter cohort compared with the reproductive cohort. In the field, non-reproductive (diapausing) summer individuals of both genders tend to be tenacious and more aggressive territorial foragers, while reproductives show mobile and less aggressive behaviour (unpublished data). Our laboratory data presented here echo this result. These behavioural differences apparently depend on the physiological (diapause) state of the animals and may be proximately controlled by developmental switches, likely yet another correlate of the all-encompassing changes known as the 'diapause syndrome' (Tauber et al. 1986). As such, they may contribute to the general environmental variance, $V_{E_{g}}$, and affect repeatability 
differentially, although it is unclear why this effect should be greater for one or the other physiological state. Greater $V_{E_{\mathfrak{g}}}$ would also probably lead to lower heritabilities, making them even harder to detect. The overall physiological state of an organism hence has to be taken into account not only in behavioural field studies but also when investigating the genetics of complex behaviour.

\section{ACKNOWLEDGMENTS}

D.P. acknowledges the German Academic Exchange Program (DAAD) and W.U.B. the NSERC of Canada for financial support. We thank C. Boake, D. Fairbairn, K. Lofdahl and R. Preziosi for valuable comments.

\section{REFERENCES}

Arnold, S. J. \& Wade, M. J. 1984. On the measurement of natural and sexual selection: applications. Evolution, 38, 709-718.

Bakker, T. C. M. 1986. Aggressiveness in sticklebacks (Gasterosteus aculeatus): a behaviour genetic study. Behaviour, 98, 1-144.

Becker, W. A. 1984. A Manual of Quantitative Genetics. 4th edn. Pullman, Washington: Academic Enterprises.

Blanckenhorn, W. U. 1991a. Fitness consequences of foraging success in water striders. Behav. Ecol, 2, 46-55.

Blanckenhorn, W. U. 1991b. Fitness consequences of food-based territoriality in water striders, Gerris remigis. Amin. Behav., 42, 147-149.

Boake, C. 1989. Repeatability: its role in evolutionary studies of mating behaviour. Evol. Ecol., 3, 173-182.

Ehlinger, T. A. \& Wilson, D. S. 1988. Complex foraging polymorphism in bluegill sunfish. Proc. natn. Acad. Sci. U.S.A., 85, 1872-1882.

Endler, J. A. 1986. Natural Selection in the Wild. Princeton, New Jersey: Princeton University Press.

Fagen, R. 1982. Evolutionary issues in development of behavioural flexibility. In: Perspectives in Ethology.
Vol. 5 (Ed. by P. P. G. Bateson \& P. H. Klopfer), pp. 365-383. New York: Plenum Press.

Fairbairn, D. J. \& Roff, D. A. 1990 . Genetic correlations among traits determining migratory tendency in the sand cricket, Gryllus firmus. Evolution, 44, 1787-1795.

Falconer, D. S. 1989. Introduction to Quantitative Genetics. 3rd edn. London: Longman.

Grant, J. A. W. \& Noakes, D. L. G. 1988. Aggression and foraging mode of young-of-the-year brook charr, Salvelinus fontinalis. Behav. Ecol Sociobiol, 22, 435-445.

Hedrick, A. V. \& Riechert, S. E. 1989. Genetically-based variation between two spider populations in foraging behaviour. Oecologia (Berl.), 80, 533-539.

Hoffmann, A. A. 1988 Heritable variation for territorial success in two Drosophila melanogaster populations. Anim. Behav., 36, $1180-1189$.

Lessels, C. M. \& Boag, P. T. 1987. Unrepeatable repeatabilities: a common mistake. $A u k, 104,116-121$.

Ketterson, E. D. \& Nolan, V. 1992. Hormones and life histories: an integrative approach. Am. Nat., 140, S33-S62.

Mousseau, T. A. \& Roff, D. A. 1987. Natural selection and the heritability of fitness components. Heredity, 59, $181-197$.

Stearns, S. C., de Jong, G. \& Newman, R. A. 1991. The effects of phenotypic plasticity on genetic constraints. Trends Ecol. Evol., 6, 122-126.

Stephens, D. W. \& Krebs, J. R. 1986. Foraging Theory. Princeton, New Jersey: Princeton University Press.

Sih, A., Krupa, J. \& Travers, S. 1990. An experimental study of the effects of predation risk and foraging regime on the mating behaviour of the water strider. Am. Nat, 135, 284-290.

Tauber, M. J., Tauber, C. A. \& Masaki, S. 1986. Seasonal Adaptations of Insects. Oxford: Oxford University Press.

Tulley, J. J. \& Huntingford, F. A. 1989. Additional information on the relationship between intra-specific aggression and anti-predator behaviour in the threespined stickleback, Gasterosteus aculeatus. Ethology, 78, 219-222.

Wearden, S. 1959. The use of the power function to determine an adequate number of progeny per sire in a genetic experiment involving half-sibs. Biometrics, $15,417-423$. 